\title{
»Une juste moitié de vos livres« Le rôle de la propagande religieuse dans la production pamphlétaire
}

L'inquiétude récurrente de la presse écrite à l'encontre du numérique, qui entraînerait la mort du livre, nous ramène aux origines de cette première révolution médiatique: celle de l'imprimé et au début d'une aventure dont on doute qu'elle prenne fin avec Internet ${ }^{1}$. Le débat sur la mort annoncée du livre remonte au moins à 1988 alors qu'Umberto Eco minimisait déjà l'impact des nouvelles technologies sur le codex qui a fait ses preuves et n'est pas prêt de disparaître (ne serait-ce que parce qu'il est malaisé d'utiliser un index sur un support numérique) $)^{2}$. La pauvreté intellectuelle de ce marronnier que rabâchent les journalistes depuis maintenant plus de vingt ans (faute de mieux) se résume dans les amalgames et anachronismes qui caractérisent le parallèle récent entre médias sociaux et imprimé ${ }^{3}$. »Ceci tuera cela«, s'exclame l'archidiacre Frollo dans »Notre-Dame de Paris« , brandissant un livre en regard de la cathédrale: »Le livre tuera l'édifice! «4 ${ }^{4}$ Pourtant le livre n'a pas tué la culture moyenâgeuse de l'image, pas plus que les révolutions numériques ne viendront à bout du livre. Le numérique, en revanche, accomplit à peu de choses près ce que l'imprimé fit pour le manuscrit: l'accélération de la reproductibilité, la diffusion exponentielle de l'information disponible et, par conséquent, la démocratisation de l'accès aux contenus. Pour terminer avec cette comparaison, il faut mentionner ici brièvement le problème de la langue, l'anglais faisant encore figure de lingua franca sur le réseau au même titre que le latin au cours du premier siècle de l'imprimé: les langues vernaculaires restant minoritaires dans les deux cas.

L'homme et la femme de la première modernité durent ressentir la même perplexité que nous face au numérique: que penser de ces artefacts, nouvellement disponibles, parfois distribués gratuitement ou placardés sur les portes des églises? Leur contenu est-il fiable? Sur quelle autorité se basent-ils? Sont-ils dangereux? Les livres sont-ils facteurs de contagion et enfin les idées nouvelles sont-elles nuisibles pour le salut de l'âme si ce n'est du corps? Autant de questions que la controverse religieuse mit en

1 Pourquoi nous n'apprendrons plus comme avant, dans: Philosophie magazine 62 (2012), p. 3557, http://www.philomag.com/archives/62-septembre-2012 (consulté le 17/07/2014).

2 Jean-Claude CARRIÈRE, Umberto ECO, N'espérez pas vous débarrasser des livres, Paris 2009.

3 Tom Standage, Writing on the Wall. Social Media. The First Two Thousand Years, Londres 2013.

4 Victor Hugo, Notre-Dame de Paris, Paris 1831, p. 5. 1. Celui-ci eu d'ailleurs un tel succès que la cathédrale qui tombait en ruine bénéficia de la notoriété du roman et fut restaurée entre 1844 et 1864 par Viollet-le-Duc. 
exergue et que les intellectuels relayèrent surtout à propos des livres de l'autre camp, les catholiques se scandalisant d'abord de l'utilisation du français vernaculaire par les réformés avant de prendre le relais dans la deuxième moitié du XVI siècle. L'inquiétude face au livre dangereux n'est pas sans rappeler notre propre panique morale face au numérique, aux »virus médiatiques«, qui engendre des théories du complot déclinées comme autant de $»$ mèmes $«^{5}$ dans tous les médias et bien sûr sur Internet, qui est le creuset de toutes les peurs, les frustrations et les cauchemars de nos contemporains. $\mathrm{Au} \mathrm{XVI}{ }^{\mathrm{e}}$ siècle comme aujourd'hui, l'analogie entre la propagation des idées nouvelles et la maladie transmise par contagion est invoquée de façon péjorative par les forces réactionnaires pour déconsidérer celles-ci.

$\mathrm{Au} \mathrm{XVI}{ }^{\mathrm{e}}$ siècle, les laïcs n'étaient pas sans guides, néanmoins, et le clergé joua ici un rôle prépondérant. Le prêtre ou le ministre était sans nul doute le premier recours pour tout chrétien confus, égaré, cherchant quelque point de référence pour se faire une idée sur les livres et libelles qui prennent une place de plus en plus importante, en France, à partir de la seconde moitié du XVI siècle. Il ne faut pas non plus oublier les diverses façons de »consommer« l'imprimé. Le livre n'était pas seulement véhicule de sens, de contenu, mais également un contenant: un objet à part entière qu'on pouvait utiliser de diverses façons, comme preuve d'appartenance à une Église ou objet de dévotion, à l'instar de la médaille d'un saint que l'on ramenait de pèlerinage par exemple ou comme un méreau qui octroyait l'entrée des temples protestants dans le Midi de la France.

Le détournement de la matérialité du livre chez des laïcs illettrés nous rappelle que l'émergence de nouveaux médias n'efface pas les modes de fonctionnement antérieurs mais viennent s'y ajouter par couches successives. L'invention de l'écriture elle-même fut décriée, dans le »Phèdre« de Platon, comme annonçant la fin de l'apprentissage "par cœur«, et par conséquent un appauvrissement du savoir humain. Le débat est remis au goût du jour chaque fois qu'une avancée technologique permet au savoir de se disséminer plus largement: l'invention de l'imprimerie et, plus récemment, la révolution numérique. Ces avancées technologiques successives permettent une extériorisation du savoir toujours plus performante, un recul critique et une historicisation du savoir toujours plus accrus qui constituent un progrès indéniable et un avantage pour les civilisations de l'écrit. C'est pourquoi, sans doute, on a tendance à associer de façon quasi systématique progrès des technologies de l'information et progrès social et politique, comme en témoigne un rapprochement hasardeux qui a été fait récemment dans l'hebdomadaire »The Economist« entre le Printemps arabe et la réforme protestante. Pour l'auteur, les Flugschriften auraient été au protestantisme ce que les médias sociaux seraient à la démocratie au Moyen-Orient: un déclencheur providentiel ${ }^{6}$. Le lien de cause à effet entre les médias sociaux et la démocratie reste à prouver et l'on

5 Le »mème« est l'équivalent médiatique du gène, concept inventé par le généticien Richard DAwKInS, The Selfish Gene, Oxford 1976.

6 Tom Standage, How Luther went viral: Five centuries before Facebook and the Arab spring, social media helped bring about the Reformation, dans: The Economist, 17/12/2011, p. 63-65; http://www.economist.com/node/21541719 (consulté le 31/03/2013). 
peut évoquer un certain nombre de contre-exemples où les nouveaux médias ont été démonisés et rendus responsables, notamment, des émeutes de Londres de l'été 2011.

En ce qui concerne le lien entre la Réforme et le livre, on peut évoquer le cas de la France où le livre imprimé servit plutôt le conformisme que la modernité et prendre en compte les impératifs économiques des imprimeurs libraires; même si le coût de production et la valeur marchande des pamphlets pouvaient paraître bien terre-à-terre aux yeux des réformateurs, pour qui l'imprimerie était d'abord une arme au service de la vraie foi. Si l'on connait bien la première réforme, dont les milliers de titres attribués à Luther témoignent encore aujourd'hui du succès, on oublie souvent la production catholique $^{7}$. Or en France, dans la seconde moitié du Xvi ${ }^{\mathrm{e}}$ siècle, elle fut indubitablement un succès et pas seulement du seul fait de la censure ou de l'appui des autorités catholiques gardiennes des privilèges éditoriaux. Les imprimeurs auraient répondu à une véritable demande, pressant les auteurs de leur fournir des textes rapidement, avec parfois des fautes. On accusait d'ailleurs les imprimeurs d'être uniquement motivés par l'appât du gain. Ce succès n'était pas du goût de tout le monde, notamment à la cour, et les imprimeurs durent parfois s'opposer à forte partie. Le libelle, et en particulier la propagande antiprotestante, prit néanmoins une place indéniable dans les choix éditoriaux et économiques des imprimeurs libraires. La production de Nicolas Chesneau, imprimeur libraire de la rue Saint-Jacques, à Paris, entre 1554 et 1583, en est un bon exemple ${ }^{8}$.

Les questions soulevées par ces choix sont multiples et les réponses ne sont pas aussi simples qu'on pourrait le croire. Le succès que rencontre le pamphlet en langue vernaculaire dans la seconde moitié du XVI ${ }^{\mathrm{e}}$ siècle en France auprès d'un »public« dont il reste à définir les contours mérite une explication qui diffère du modèle normatif de la réforme luthérienne. D'ailleurs comme l'a fort bien démontré Tatiana Debbagi Baranova, l'imprimé répond principalement à une logique de justification après coup, ou de légitimation en préparation à une prise de position politique, dans un espace public déjà conquis. Contrairement à l'idée reçue d'une sphère publique en gestation, dont les réformes auraient été le creuset, l'opinion publique au XVI siècle se prête à des interprétations tout autres. Conformément à une interprétation antique et médiévale, l'opinion du plus grand nombre était un vecteur de surnaturel (vox populi vox dei disaient les contemporains) qui s'oppose à la raison de l'homme public qui doit rendre compte du bien commun par le jugement ${ }^{9}$. Cette opposition se retrouve dans les formes

7 À ce titre John Frymire propose une réévaluation de la contribution catholique dans l'Empire lors de la réforme luthérienne, notamment en ce qui concerne les recueils de sermons; John M. FRYMIRE, The Primacy of the Postils. Catholics, Protestants and the Dissemination of Ideas in Early Modern Germany, Londres 2010 (Studies in Medieval and Reformation Traditions, 147).

8 Luc RaCAut, Nicolas Chesneau. Catholic Printer in Paris during the French Wars of Religion. 1558-1584, dans: Historical Journal 52 (2009), p. 23-41.

9 Sandro LANDI, art. »Opinion publique«, dans: Olivier Christin (dir.), Dictionnaire des concepts nomades en sciences humaines, Paris 2010, p. 363-382. 
formes d'argumentation rhétorique, opposant raison et passion, que l'on retrouve dans les libelles ${ }^{10}$.

On peut convenir qu'il y a un public et qu'il a une opinion mais il serait néanmoins abusif de faire des réformes le berceau de »l'opinion publique« telle qu'Habermas a pu la définir, conçue à partir du XVIII ${ }^{\mathrm{e}}$ siècle comme ultime caution des décisions politiques par le biais du suffrage. Rappelons simplement pour dissiper ce faux semblant qu'au $\mathrm{XVI}^{\mathrm{e}}$ siècle »opinion« est un terme péjoratif souvent employé au pluriel pour désigner les erreurs du parti contraire. Mais force est de constater que jusqu'au milieu du XVI ${ }^{\mathrm{e}}$ siècle en France, la principale clientèle du livre imprimé en latin est un parterre de lettrés composé de clercs, de maîtres d'école et d'université, d'étudiants, et de juristes. Or, vers 1550, la production, en terme de nombre d'éditions brut, prend un virage décisif, privilégiant les pamphlets en langue vernaculaire qui cherchent, non pas à convertir les lecteurs aux nouvelles opinions, mais, a contrario, à les en éloigner. L'évolution politique du royaume, à la suite de la mort d'Henri II, suscite bien entendu une mobilisation croissante des partis autour du protestantisme et de ses opposants. Les pics de production, en 1561 et 1588 , correspondent bien aux deux principales crises que connaît alors le royaume: le début des guerres de Religion et la Ligue. La mobilisation croissante de l'opinion du plus grand nombre appelée à juger de la fama (réputation) des chefs de partis, élevée au statut de preuve dans une argumentation quasi judiciaire de la validité d'une cause ou d'une autre rendrait en partie compte de cette courbe ${ }^{11}$.

Néanmoins, une part non négligeable de cette production concerne l'éducation des laïcs, avec la multiplication d'arguments qui mobilisent tour à tour la passion (dans un sens positif) et la raison des lecteurs catholiques pour la sauvegarde de l'Église menacée par le protestantisme, bien sûr, mais aussi par la couronne qui la tolère. Cette production répondrait en partie à l'injonction du concile de Trente de pourvoir à l'éducation des laïcs en langue vernaculaire par la publication de catéchismes et de livres de dévotion mais pas uniquement: une autre partie de cette production répond point par point aux arguments des réformés, au risque d'encourager la haine. L'année 1561 en particulier témoigne du foisonnement des tactiques employées pour dénoncer l'hérésie, sans grande cohérence, qui dénote une période expérimentale non dénuée d'une certaine précipitation, peut-être en réponse à la politique de conciliation de Catherine de Médicis. Cette effervescence ne peut pas s'expliquer par l'offre seule, auteurs et imprimeurs se méfiaient du pamphlet et lui préféraient les beaux livres destinés à un public lettré, mais aurait bien répondu à une demande du »public«. Si les imprimeurs parisiens répondent à une véritable demande, doit-on conclure pour autant,

10 Tatiana DebBagi Baranova, À coups de libelles. Une culture politique au temps des guerres de Religion (1562-1598), Genève 2012 (Cahiers d'humanisme er Renaissance, 104), p. 342 344.

11 Thelma S. Fenster, Daniel Lord Smail (dir.), Fama. The Politics of Talk and Reputation in Medieval Europe, Ithaca 2003. 
comme le fera la propagande royaliste après la Ligue, que c'est le clergé qui attise les passions des laïcs ${ }^{12}$ ?

Or le clergé lui-même est divisé autour de cette question: faut-il ou non instruire le peuple des opinions de l'adversaire protestant afin de l'éduquer ou simplement lui rappeler ce en quoi il doit croire pour atteindre le salut de son âme? Cette question préoccupe tellement les ecclésiastiques catholiques qu'elle est évoquée explicitement lors de la dernière session du concile de Trente par l'archevêque de Rossano en 1562:

La clarté des canons rend inutile la doctrina. Et d'ailleurs à qui s'adresse-t-elle? Si elle vise les hérétiques, les arguments qu'elle met en œuvre sont précisément ceux que ces derniers récusent Si la doctrina est rédigée pour les catholiques, n'ont-ils pas pour s'instruire la liturgie de la messe elle-même, le canon et les autres prières ${ }^{13}$ ?

Les auteurs catholiques étudiés ici sont divisés, et changent parfois de façon de penser alors que l'évolution de la situation politique en France les pousse à adopter des positions qu'ils n'auraient peut-être pas choisies par eux-mêmes. Gentian Hervet dans son catéchisme de 1561 résume de façon succincte le dilemme auquel les réformateurs catholiques durent faire face dès le début de la pénétration des idées protestantes en France. Les analogies entre l'hérésie et la maladie ou le feu, toutes deux sources d'une anxiété tout aussi contagieuse que leur objet dans la France de la première modernité, fusent tout comme les recettes pour endiguer l'hérésie:

Avons nous pas dict que l'heresie est comme un feu? Voyons doncques si comme on estainct le feu, il est possible de l'estaindre. Comment doncques estainct lon le feu? Il y a deux moyens: l'un est d'aller au devant, avecques eau froide \& vinaigre, \& toutes choses qui sont excessivement froides. L'autre, c'est d'oster au feu le nourrissement, \& specialement les matieres lesquelles sont aisees à allumer, comme chaulme, paille, souffre, huille, \& choses semblables. Or regardons si ce n'est pas le mesme moyen d'estaindre l'heresie ${ }^{14}$.

On peut rapprocher ce passage des deux modes d'argumentations rhétoriques identifiés par Debbagi Baranova, sacré et judiciaire, le premier s'adressant à un lecteur déjà acquis et le second à tous les lecteurs, y compris ceux qui doutent ou qui ont déjà basculé dans l'hérésie $^{15}$. Les auteurs adoptent tour à tour ces stratégies, qui peuvent paraître contradictoires, parfois au sein d'un même ouvrage ou d'une collection, invitant une lecture à plusieurs niveaux. On peut citer à cet égard l'auteur en langue française le plus prolifique de la seconde moitié du XVI ${ }^{\mathrm{e}}$ siècle (dont le catalogue compte 228 notices) ${ }^{16}$ : le curé de Saint-Eustache, René Benoist, surnommé le »pape des

12 Mark Greengrass, Governing Passions. Peace and Reform in the French Kingdom. 15761585, Oxford 2007.

13 Cité d'après André Duval, Des sacrements au concile de Trente, Paris 1985, p. 99.

14 Gentian HeRvet, Epistre ov advertissement av pevple fidele de l'Eglise Catholique. Touchant les differens qui sont aujourd'huy en la religion Chrestienne, Paris 1561, fol. $3 \mathrm{v}$.

15 DebBagi Baranova, Â coups de libelles (voir n. 10), p. 453.

16 Andrew Pettegree, Malcolm Walsby, Alexander Wilkinson (éd.), French Vernacular Books. Books Published in the French Language before 1601, vol. I, Leyde 2007, p. 101-108. 
Halles« autant pour ses talents de prêcheur que pour sa Bible en français, qui lui attira les foudres de la Sorbonne. Lors d'un sermon prononcé à la Toussaint en 1561, Benoist s'adresse ainsi à son auditoire:

Toutesfois qui vouldroit bien rechercher le nombre de ceux qui demeurent fermes en la foy [...] il seroit trouvé beaucoup plus grand que celuy de ceux qui tiennent le contraire [...] lesquelz selon la parole de Dieu sont instruictz \& confortez en la verité de la foy par le S. esprit, asçavoir les simples \& pauvres ${ }^{17}$.

On peut comparer cette remarque avec ce que dit Gentian Hervet plus tard, en 1568, à propos d'un catéchisme que le cardinal Charles de Lorraine lui avait commandé avant leur départ pour le concile de Trente, en 1561:

Monseigneur le Cardinal de Lorraine [...] non content de la bonne instruction qu'il donne au peuple [...] fait composer un petit Catechisme pour son Diocese, duquel le simple populaire, qui n'est pas curieux d'entrer aux questions par trop hautes \& subtiles, n'en peult estre sinon satisfait. Mais depuis considerant que le peuple estoit devenu un peu plus curieux que de constume, \& qu'il ne luy suffisoit pas de croire, mais demandoit encores quelques raison de sa foy, afin de pouvoir plus aiséement resister aux heretiques: \& pourtant desirant qu'il se fist un Catechisme un peu plus ample, me declara quelque temps devant qu'il s'en allast au Concile, \& me dist qu'il eust bien voulu que j'eusse entrepris cette charge ${ }^{18}$.

On retrouve donc l'opposition entre une exposition simple de la foi destinée à un auditoire acquis et instruit par le Saint-Esprit et une explication par le menu de la doctrine chrétienne telle qu'elle est redéfinie lors du concile de Trente. Alors que Benoist en 1561 semblait confiant dans l'orthodoxie de ses paroissiens, en 1569 il s'adresse aux paroissiens de Saint-Eustache »ayans este seduicts et trompez sous couleur et pretexte d'une Eglise reformee [...] se sont retranchez de [...] l'Eglise catholique« et en 1573 il propose un catéchisme "pour l'instruction de ceux qui ont esté mal instruicts \& catechisez par les hérétiques «19. On trouve d'autres indices de la tension entre ces deux

17 René Benoist, Manifeste et necessaire probation de l'adoration de Jesus Christ, Dieu \& homme en l'Hostie sacrée, tant en la Messe que en tout autre lieu auquel elle est presentée aux Chrestiens, Paris 1562 , fol. $25 \mathrm{v}$.

18 Gentian HeRveT, Catechisme, et ample instrvction de tovt ce qui appartient av devoir d'vn chrestien principalement des Curez \& Vicaires, \& tous ceux qui ont charge des Eglises Parrochiales, en ce qui est requis au principal deuoir de leurs charges. Auec responses à tout ce qu'obiectent les heretiques, tant contre les Sacremens, qu'autres choses qui concernent la foy $\&$ religion de l'Eglise Catholique. Faict pour l'instruction du simple peuple, selon le commandement de Mon-seigneur le Cardinal de Lorraine, Paris 1568 [épître non paginée de l'auteur à Renée de Lorraine, 16 septembre 1567], p. iv-v.

19 René BENOIST, Advertissement exhortatoire a ceux de la parroisse de S. Eustache à Paris, lesquels ayans esté seduicts \& trompez sous couleur \& pretexte d'vne Eglise reformee \& plus pure religion, se sont retranchez de la profession de la Foy \& Religion Chrestienne, proposee en l'Eglise Catholique, hors laquelle il n'y a point de salut., Paris 1569; ID., Catecheses ov instrvctions touchant les poincts à present controuerses en la religion, ac commodées aux Euangiles d'vn chacun iour de Caresme: proposées en sermons en l'Eglise de S. Eustache à Paris, l'An 1573, pour l'instruction de ceux qui ont esté mal instruicts \& catechisez par les heretiques, Paris 1573. 
impératifs - l'instruction de ceux acquis à la vraie foi et la persuasion des indécis dans la politique éditoriale de Nicolas Chesneau qui s'adresse ainsi à deux »publics« distincts, mettant à mal l'efficacité du discours. Les imprimeurs libraires devaient prendre en compte d'autres facteurs que la simple efficacité et ceux-ci étaient, malgré l'appui des mécènes, sujets à la pression du marché.

Une bonne illustration de ce phénomène est l'édition des sermons de François Le Picart, tels qu'ils avaient été prêchés, comme si ce fait constituait à lui seul un cachet d'authenticité. Ainsi, l'édition des sermons établie par Benoist inaugure une longue collaboration entre ce dernier et Chesneau qui ne s'achève qu'à la mort de celui-ci en 1583 et qui est à l'origine de pas moins de soixante-cinq éditions en français vernaculaire, sans compter les vingt-sept titres de l'édition des sermons de Le Picart ${ }^{20}$. Celle-ci est publiée en plusieurs volumes entre 1560 et 1571, et mentionne encore sur toutes les pages de titre »Fidelement recueilliz, ainsi qu'ils ont esté prononcez«. Dans une lettre dédicatoire, Benoist nous donne un indice precieux sur cette collaboration, rappelant que les sermons du célèbre prédicateur catholique avaient été rapportés de première main par un témoin oculaire:

Pourquoy, j'ay cherché autres moyens de m'asseurer en cest endroit: à quoy je confesse libremement, que la fidelité, prudence \& curiosité de son honneur, du sire Nicolas Chesneau (lequel comme volontiers fait imprimer les livres, lesquels il cognoist prouffitables \& necessaires à la Republique chrestienne, aussi ne propose il rien legerement, sans estre bien asseuré) par la diligence duquel iceux Sermons ont esté cherchez, reveuz, corrigez, \& imprimez, m'a beaucoup confermé iceux sermons estre de cestuy, au nom duquel ils sont publiez. Mais voulant estre du tout certioré de la verité, pour desormais ne douter en rien de cest affaire, me sui addressé audit Chesneau, \& usant de la familiere amitié, laquelle de long temps a esté reciproque entre nous deux, ay voulu sçavoir au vray si iceux sermons estoient de cestuy auquel il les attribuoit. Alors il m'a declaré entierement, comment il les avoit retirez par le moyen de ceux lesquels ordinairement suyvoient iceluy docte personnage, duquel ils escrivoient autant diligemment qu'il leur estoit possible, les sermons, sans changer (sinon bien peu, si cela advenoit quelques fois) ny sentence ny parole. Iceluy Chesneau, aussi souvent m'a monstré divers exemplaires d'iceux sermons, recueillis par plusieurs, lesquels avoit esté frequens auditeurs, \& avoir assidument escrit \& receuilly les sermons dudit seigneur Picart, l'on ne sçauroit nier. Et quant à moy, cela m'a beaucoup asseuré qu'il m'a nommé et affermé luy avoir baillé tels sermons, ceux que je sçavois y avoir veu souvent ${ }^{21}$.

On ne peut que répéter la prépondérance de l'oral sur l'écrit à cette époque, que les imprimeurs mêmes reconnaissent et mettent en avant comme argument de vente. Pourtant l'œuvre ne semble pas avoir eu tout le retentissement que Chesneau ou Benoist

20 Pettegree, Walsby, Wilkinson (éd.), French Vernacular Books (voir n. 16).

21 François LE PICART, Les sermons et instrvctions Chrestiennes, pour tous les iours de Caresme, $\&$ Feries de Pasques. Enrichiz d'vn sermon pour le iour \& feste de l'Annonciation de la vierge Marie. Fidelement recueilliz, ainsi qu'ils sont esté prononcez par feu de bonne memoire, Monsieur nostre Maistre François le Picart, Docteur en Theologie à Paris, Paris 1566 [épître non paginée de René Benoist aux habitans de Paris, 20 décembre 1563], p. iii-iv; Larissa TAYLOR, Heresy and Orthodoxy in Sixteenth-Century Paris. François Le Picart and the Beginnings of the Catholic Reformation, Leyde 1999, p. 1-5; RACAUT, Nicolas Chesneau (voir n. 8), p. 31. 
auraient pu escompter, vu la notoriété de Le Picart, et l'imprimeur fait bientôt appel à des mécènes pour la continuation de cette entreprise ambitieuse. Ainsi Chesneau s'adresse-t-il à Renée de Lorraine, abbesse de Reims, en 1563:

qu'ayant ja dedié à vostre bonté \& grandeur deux tomes du recueil de ses sermons, \& ayant mis toute peine $\&$ diligence à moy possible de revoir \& collationner sur plusieurs originaux, \& de faire correctement imprimer (jaçoit que n'ait esté si tost que desirois à cause de la malice du temps) ces presens sermons ${ }^{22}$.

En effet, l'édition complète des sermons de Le Picart en quatre volumes in-octavo à Paris s'effectue en parallèle avec les imprimeurs du cardinal de Lorraine à Reims, dont le Picart était un des maîtres à penser. C'est une entreprise coûteuse qui s'étale sur plus de dix ans et qui n'eût peut-être pas vu le jour sans le mécénat des Guises. Il serait hasardeux de juger de son succès d'après le nombre d'exemplaires qui ont survécu en se basant sur le décompte établi par les »Livres vernaculaires français«, mais on peut émettre l'hypothèse que ce ne fut pas un best-seller malgré la notoriété posthume de Le Picart. Chesneau, par ailleurs, donne une indication supplémentaire de la distinction qu'il fait entre les livres, qu'il estime être méritoires, et les libelles, dont il publie pourtant lui-même un certain nombre, dans une épître que l'imprimeur adresse à Pierre Rousseau, chanoine d'Angers et prévôt de Saint-Laurent en 1566:

Je vous ay choisi entre plusieurs, pour soubs l'authorité de vostre nom, mettre en lumiere cest euvre, non de telle quelle literature \& matiere, que sont en nostre temps plusieurs escrits, qui ne meritent encherir le papier, \& faire perdre le temps à plusieurs personnes, mais grave \& traittant choses necessaires à tout homme pour estre sauvé23.

J'attire ici l'attention sur le fait que Chesneau feint de voir d'un assez mauvais œil certains livres qui d'après lui ne valent pas le papier sur lequel ils sont imprimés. Il y a là une contradiction entre le ton qu'emploie Chesneau lorsqu'il s'adresse aux mécènes et celui qu'il emploie pour s'adresser au public auquel ses livres sont destinés. On peut dire que Chesneau joue sur les deux tableaux: d'une part en fustigeant les brûlots pour s'attirer le mécénat et les bonnes grâces des puissants afin de produire des livres dignes d'un grand imprimeur, et d'autre part en produisant lui-même une partie non négligeable de ces brûlots en s'adressant à son lectorat plus humble en des termes autrement plus relevés ${ }^{24}$. Il est indéniable que l'imprimeur parisien bénéficie du mécénat des Guises, notamment pour les éditions des multiples catéchismes et livres d'instruction

22 LE PICART, Les sermons (voir n. 21) [épître non paginée de Nicolas Chesneau à Renée de Lorraine, 20 décembre 1563], p. ii.

${ }^{23}$ ID., Les sermons, et instructions Chrestiennes, pour tous les jours de l'Advent, jusques à Noel: $\&$ de tous les Dimenches \& Festes, depuis Noel jusques à Caresme. Enrichis de quatre sermons non encore par cy devant imprimez: desquels est faicte mention en la page suyvante. Fidelement recueillis, ainsi qu'ils ont esté prononcez, par feu de bonne mémoire, Monsieur Maistre François le Picard, Docteur en Theologie à Paris, Paris 1566 [épître non paginée de Nicolas Chesneau à Pierre Rousseau, 10 novembre 1564], p. iii.

24 RACAUT, Nicolas Chesneau (voir n. 8). 
des laïcs, qui sont le fruit des efforts prétridentins du cardinal de Lorraine, ou l'édition complète des décrets et canons du concile de Trente en français. Par ailleurs, Chesneau fait son apprentissage dans l'atelier de Claude Frémy en publiant des brûlots qui participent à la campagne antiprotestante menée par des catholiques intransigeants au tournant décisif des années 1560-156425.

L'année 1561 est particulièrement significative et constitue un pic de production de l'imprimeur, au mitan entre l'explosion pamphlétaire liée à la première réforme luthérienne et le second pic de production lié au double assassinat des Guises et à l'occupation de Paris par la Ligue ${ }^{26}$. Un recueil de textes de cette année est remarquable de par son hétérogénéité et son succès que l'on peut déduire du nombre impressionnant de rééditions, variations de la page de titre et tirages simultanés sur plusieurs presses parisiennes (au moins trois). Il s'agit des »Contrarietez et contradicts qui se trouvent en la doctrine de Jean Calvin« d'Antoine Duval, un recueil in-octavo de pièces courtes destinées aux laïcs attaquant de front la théologie réformée 27. L'ouvrage est un recueil de quatre livres distincts, traduits d'auteurs étrangers: le premier est une réfutation point par point de la théologie protestante organisée en une table, le second s'attache à la question de la prédestination et le troisième est un résumé de la défense du libre arbitre d'Érasme. La quatrième pièce, rapportée il semblerait à la dernière minute, est un catéchisme de Guillaume Lindan, évêque de Ruremonde, que Gentian Hervet a traduit pour l'occasion, mais qui ne figure pas, de façon significative, dans les éditions suivantes de la compilation. Il est intéressant de noter que la deuxième pièce, les »Demandes et Repliques à Jean Calvin« cite deux éditions distinctes des »Instituts de la Religion Chrétienne« (celles de 1545 et 1557) afin de démontrer les incohérences internes dans la doctrine de Calvin mais également les différences avec le Luthéranisme. Il paraît judicieux de noter qu'en 1561, bien que la confessionnalisation soit déjà en cours, les catholiques français ne font pas encore très bien la différence entre luthériens et calvinistes.

La première pièce, les »Contrarietez et contradicts«, qui donne son titre à la compilation, est d'autant plus remarquable qu'elle emploie un artifice visuel mettant côte à côte les diverses positions luthériennes, calvinistes et catholiques, sur une même page. Par exemple, la proposition »le Christ n'est pas présent en l'Eucharistie« figure à coté d'autres propositions présentées de gauche à droite en tableau avec l'interprétation »correcte« (catholique) à droite. L'intention pédagogique de l'éditeur et du traducteur est évidente: permettre au lecteur de »cognoistre au doigt et voir à l'œil« les erreurs

25 Luc Racaut, Hatred in Print. Catholic Propaganda and Protestant Identity during the French Wars of Religion, Aldershot 2002.

26 Andrew PetTegree, Reformation and the Culture of Persuasion, Cambridge 2005, p. 177-184.

27 Antoine Duval, Les contrarietez et contradicts, qui se trovvent en la doctrine de Iean Caluin, de Luter \& autres nouueaux euangelistes de nostre temps. Auec es demandes \& repliques à Iean Caluin sur son liure de la Predestination. Vn recueil d'aucuns escrites d'Erasme contre les Luteriens. Vn Catechisme ou sommaire de la foy \& deuoir du vray Chrestien, contre les heresies de ce temps [...], Paris 1561. 
des protestants, avec la bonne interprétation en dernière colonne ${ }^{28}$. Les propositions ainsi présentées constituent, sur neuf pages in-octavo, une défense élémentaire des sacrements, des bonnes œuvres, de la doctrine du libre arbitre et du purgatoire. C'est un exemple exceptionnel des efforts pédagogiques des catholiques pour éduquer les laïcs, les informant par le même biais des différences entre la doctrine catholique et celle des réformés. Il semblerait que les »Contrarietez et contradicts« aient aussi été un succès éditorial, dans la mesure où l'on peut en juger par les variations des pages de titre, les rééditions et le nombre d'exemplaires qui ont survécu. La version qui comprend le catéchisme de Lindan est particulièrement intéressante, car l'éditeur, Duval, nous donne des indications précieuses sur la façon dont la compilation a été conçue.

En effet, comme les autres pièces de la compilation, le catéchisme de Lindan existe en tant qu'édition à part entière avec sa page de titre propre ${ }^{29}$. Une courte épître est insérée à la fin de la troisième pièce, indiquant comment le catéchisme fut inclus dans la collection après coup alors qu'il était destiné à être imprimé séparément:

Ayant doncques communiqué avec l'Imprimeur, pour imprimer les contrarietez susdictes, \& luy ayant laissé nostre copie en main pour veoir, il nous en a communiqué une autre, fort propre \& convenable à ce que dessus: laquelle luy avoit esté baillée pour imprimer. L'ayant doncques veue $\&$ trouvée avoir esté extraicte de l'oeuvre mesme duque avons recueilly les contrarietez de Luther, $\&$ autres, avons (comme de raison) acquiescé à la requeste dudict Imprimeur ${ }^{30}$.

C'est donc l'imprimeur qui propose à Duval d'insérer le catéchisme de Lindan dans la collection. L'imprimeur participe ainsi à l'élaboration des œuvres qu'il produit, s'élevant donc au-dessus du statut de simple artisan exécutant une commande. Il est probable que la personne qui avait envoyé le catéchisme à Chesneau ait été Gentian Hervet, qui l'avait traduit, ou même le cardinal de Lorraine, qui, on l'a vu, était attaché à l'éducation des laïcs. Le plus étonnant est ce qu'écrit Duval pour justifier l'inclusion d'un catéchisme qui s'adresse principalement à ceux qui était acquis à la cause catholique, côte à côte avec des écrits qui sont plutôt destinés aux indécis:

Aussi (par l'advis mesme de plusieurs) ne nous a semblé avoir assez faict, d'avoir monstré au doigt les contrarietez contenues es livres \& fausse doctrine de Calvin, de Luter, Melanchthon, \& autres tels reveres personnages de nostre temps, si nous ne donnions (aux simples \& illiterez principalement) la medecine: \& adjoustant ce qu'il fault que tout Chrestien tienne \& croye au contraire, sur peine de damnation eternelle ${ }^{31}$.

28 Ibid., fol. 2v, 25v, »Traicte en Manière de Table recueilly des œuvres de Guillaume Lindan«.

29 Willem Lindanus, Gentian Hervet (trad.), Catechisme ou sommaire de la foy, Paris 1561.

30 Duval, Les contrarietez et contradicts (voir n. 27), »Antoine du Val au lecteur« non paginé. La pagination et les signes typographiques sont erronées à partir du fol. 41, sig. F1 dans l'exemplaire conservée à la Bibliothèque nationale de France, cote D-33378 (1), et disponible en ligne sur http://eeb.chadwyck.co.uk/ (consulté le 07/08/2014). La lettre est une préface du »Catechisme ou sommaire de la foy«, cote D-33378 (3) mais précède »Les demandes et répliques à Jean Calvin«, cote D-33378 (2).

31 Ibid. 
Le catéchisme s'apparente en effet au premier groupe de textes, de type sacré, emportant l'adhésion des catholiques déjà acquis, alors que les autres textes s'apparentent plutôt à l'argumentation judiciaire, faisant appel à la raison du lecteur pour convenir des erreurs des hérétiques. Chesneau aurait ainsi voulu concilier les deux approches en un seul recueil. Le fait que l'épître ait été insérée entre le troisième texte de la collection pour justifier cette démarche indique que c'était peut-être là une décision de dernière minute. Pourquoi cette décision de la part de l'imprimeur? Le catéchisme aurait eu une distribution plus large en recueil plutôt que vendu séparément et Chesneau voulait ainsi maximiser tout simplement la diffusion de cet ouvrage. Le recours à l'analogie avec la maladie dans cette même épître est aussi révélateur d'une volonté d'équilibrer les deux démarches, et de s'adresser à la fois à ceux qui était déjà acquis au catholicisme et aux indécis:

Comme ce n'est assez au medecin de dire au malade, mon amy ou mamie, vous avez faict exces, vostre mal vous tient là ou vous estes bien malade: Mais il fault après avoir cognoissance, tant de la maladie que de la cause d'icelle, enseigner et applicquer la medicine propre pour couvrir la sante, \& chasser la maladie ${ }^{32}$.

Dans son catéchisme de 1568, Gentian Hervet reprend à peu près les mêmes termes:

En cecy certes, nous voyons bien que la regle des medecins est veritable, qui dit: que le contraire est curé par son contraire. Car comme l'epidimie d'heresie n'est procedée d'autre chose que de la doctrine pestilente contenue aux Catechismes des modernes heretiques, aussi le souverain remede pour y obvier, est de bailler la contrepoison, avecques les Catechisme de l'Eglise Catholique ${ }^{33}$.

Nous trouvons d'autres exemples de l'embarras que ce mélange des genres pouvait susciter alors que les auteurs, souvent entraînés dans des échanges ad hominem contre leur gré, doivent se justifier de défendre leur honneur ainsi que celui de la vraie foi. Les libelles diffamatoires étaient perçus comme un mal nécessaire - les prêtres en tant que personnages publics devaient se prémunir des attaques de leurs adversaires - mais préjudiciable à l'éducation des laïcs. Pourtant, compte tenu du succès que ce genre remporte dans la seconde moitié du $\mathrm{XVI}^{\mathrm{e}}$ siècle, il semblerait qu'il était plus vendeur que les ouvrages de dévotion et d'instruction des laïcs. Et cela malgré les interdits royaux contre l'invective, réitérés par tous les édits de pacification de 1562 jusqu'à 1598.

Les auteurs se défendent de vouloir jeter de l'huile sur le feu tout en reportant la responsabilité sur leurs adversaires. On peut citer à cet égard l'échange entre Jean de L'Espine et René Benoist au cours duquel L'Espine attribue la prodigalité de Benoist à la médiocrité des textes de ce dernier, rédigés à la hâte:

Aussi te diray-je, qu'il ne faut point, que pour monstrer la dexterité de ton esprit, \& acquerir quelque lieu entre les gens de sçavoir, tu te vantes d'avoir composé ton livre en peu de jours: car

32 Ibid.

33 Hervet, Catechisme (voir n. 18), p. iv. 
nous ne nous estonnerions pas quand en moindre temps que tu dis, tu en aurois composé une douzaine de pareils ${ }^{34}$.

De plus, L'Espine attribue la précipitation avec laquelle il a dû lui-même rédiger ses propres réponses à la cupidité des imprimeurs libraires qui le presse de fournir des textes rapidement, au risque de laisser des fautes, par simple appât du gain:

Toutesfois il en est advenu tout autrement, \& que contre mon desir il a esté imprimé assez negligemment, dont il me deplaist fort, \& que par la precipitation \& cupidité des Libraires \& Imprimeurs, les mysteres de nostre religion ne sont maniez avec une plus grande attention \& reverence 35

L'état de précipitation dans lequel se trouvent les imprimeurs, qu'ils transmettent aux auteurs, est donc bien réel et procède d'une pression qu'on peut attribuer au succès que remportent les libelles auprès du public ${ }^{36}$. Il est intéressant de noter que Benoist ne se gêne pas pour faire porter à son tour la responsabilité des fautes que sa Bible pourrait contenir à l'imprimeur tandis que sa publication en français en 1566 fait scandale et suscite le courroux de la Sorbonne:

Pourquoy je pense bien qu'il s'en trouvera quelques uns qui se sentiront offencez de peu de chose: comme si par quelque faulte d'aucuns imprimeurs (lesquelz quand ilz veulent estre mauvais $\&$ infideles, font plus de mal, \& donnent plus de fascherie que je vouldroye n'en avoir experimenté) un mot n'est si bien couché, une ligne n'est si bien tyree, un chiffre \& cottation mise pour l'autre, \& ainsi des aultres petites fautes ${ }^{37}$.

Si c'était le cas pour la Bible, ce devait être d'autant plus vrai pour les libelles et L'Espine insiste sur la nature bon marché et par conséquent de mauvaise qualité des livres de Benoist:

34 René BENOIST, Brieve et facile refutation d'un livret divulgué au nom de I. de l'Espine, se disant Ministre de la parole de Dieu:auquel violentant \& detorquant l'escriture saincte, il blaspheme malheureusement le sainct sacrifice Euãgelique, dict vulgairement la saincte Messe, Paris 1564; Jean de L'ESPINE, Defense et confirmation du traicté du vray Sacrifice \& Sacrificateur, faict par M. Iehan de l'Espine Ministre de la parolle de Dieu, A l'encontre des friuoles responses \& argumens de M. René Benoist, Angeuin, Docteur en Theologie, Geneve 1567, p. 6-7; Luc RaCAUT, Education of the Laity and Advocacy of Violence in Print during the French Wars of Religion, dans: History. The Journal of the Historical Association 95 (2010), p. 159-176, ici p. 160.

35 L'ESPINE, Defense et Confirmation (voir n. 34), p. 2.

36 Jean-François GiLmONT, Le livre et ses secrets, Genève 2003, p. 154.

37 René BenOIST, La Sainte Bible Contenant le Vieil \& Nouueau Testament, Traduitte en François, selon la version commune: Auec annotations necessaires pour l'intelligence des lieux les plus difficiles: \& expositions contenantes briefues \& familieres Resolutions des lieux qui ont esté depraués \& corrompus par les heretiques de nostre temps: Aussi les figures \& argumens sur chacun liure, declarans sommairement tout ce que y est contenu. Par M. René Benoist, Angeuin, Docteur Regent en la faculté de Theologie à Paris, Paris 1566 [Advertissemens apologetiques non paginés, $\left.n^{\circ} 6\right]$, p. iii. 
Tes livres sont comme ces menues merceries, qu'on fait \& vend à la douzaine, \& une marchandise telle qu'il fault aux petis col-porteurs du Palais: car ils ont besoin aussi bien que les vins de petit pris, d'un crieur babillard \& affeté pour les vendre \& debiter 38 .

Il est intéressant de noter ici le rôle qu'attribue L'Espine au prix bas du livre et l'influence néfaste sur les consciences qu'il y associe. Le livre publié à la va-vite et vendu à petit prix sert, d'après lui, de plate-forme aux prédicateurs catholiques pour inciter le peuple à la haine des protestants:

Or ayant esté ledict traicté mis en lumiere, je fu adverti que ceux qui preschoyent lors à Paris s'en alteroyent fort, \& qu'à leur maniere accoustumee ils employoyent la pluspart de leurs sermons en invectives, brocards \& injures contre moy ${ }^{39}$.

Et L'Espine conclut sa réponse à Benoist, avec une estimation de la place des libelles dans la production catholique qui mérite qu'on s'y arrête, de par son apparente acuité: »Et finalement tant de brocards, injures \& blasons, qui contiennent \& comprennent pour le moins une juste moitié de vos livres $«^{40}$. Si cette estimation est hasardeuse, elle reflète néanmoins un état de fait que les imprimeurs et les auteurs eux-mêmes reconnaissent: tous les livres ne se valent pas. Beaucoup estiment pourtant qu'il est nécessaire pour combattre l'hérésie de donner dans les deux registres: l'instruction des laïcs acquis au catholicisme par le biais de catéchismes et d'œuvres pastorales d'une part, et l'argumentation raisonnée pour convaincre les indécis qui passe souvent par une déconsidération polémique de l'ennemi protestant d'autre part.

Ces deux impératifs sont contrastés par les auteurs eux-mêmes, mais, comme on a pu le voir dans les »Contrarietez et contradicts«, ils peuvent cohabiter au cœur d'un même recueil et parfois au sein d'un même ouvrage. Les rares observations qui ont trait aux aspects matériels de la production du livre imprimé, son coût et sa valeur marchande, nous invitent par ailleurs à considérer l'imprimeur libraire comme un agent historique à part entière. Même si les imprimeurs pouvaient bénéficier de l'aide de mécènes, il ne faut pas oublier la dimension mercantile de leur entreprise: les imprimeurs libraires étaient tributaires de facteurs économiques aussi bien que spirituels, et ces premiers ont pu agir comme des freins ou des accélérateurs à la réforme catholique.

\section{RÉSUMÉ}

En ce qui concerne le lien entre la Réforme et le livre, on peut évoquer le cas de la France - où le livre imprimé servit plutôt le conformisme que la modernité - et prendre en compte les impératifs économiques des imprimeurs libraires, même si le coût de production et la valeur marchande des pamphlets pouvaient paraître bien terre à terre aux yeux des réformateurs, pour qui l'imprimerie était d'abord une arme au service de la vraie foi. Si l'on connaît bien la première réforme - les

\footnotetext{
38 L'EsPINE, Defense et Confirmation (voir n. 34), p. 7.

39 Ibid., p. 2.

40 Ibid., p. 94.
} 
milliers de titres attribués à Luther témoignent encore aujourd'hui de son succès -, on oublie souvent la production catholique. Or, en France, dans la seconde moitié du XVI ${ }^{\mathrm{e}}$ siècle, elle fut indubitablement très en vogue, et pas seulement du seul fait de la censure ou de l'appui des autorités catholiques, gardiennes des privilèges éditoriaux. Les imprimeurs auraient répondu à une véritable demande, pressant les auteurs de leur fournir des textes rapidement, même s'ils contenaient parfois des fautes. (Les imprimeurs étaient accusés d'être uniquement motivés par l'appât du gain.) Ce succès n'était pas du goût de tout le monde, notamment à la cour, et les imprimeurs durent parfois s'opposer à forte partie. Le libelle, et en particulier la propagande antiprotestante, prit néanmoins une place indéniable dans les choix éditoriaux et économiques des imprimeurs libraires.

\section{SUMMARY}

In the case of France, the printed book rather served conformism than modernity as far as the association between reformation and the book is concerned. It is necessary to take the economic priorities of printer-booksellers into account, even though production costs and the mercantile value of pamphlets could appear quite trivial in the eyes of reformers for whom printing was, first of all, a weapon serving the true faith. The thousands of titles attributed to Luther attest even today to the success of the Reformation, while the Catholic production is often forgotten. In France, however, in the second half of the sixteenth century, the latter was without doubt successful, and this not only due to the mere fact of censure or the support of Catholic authorities guarding editorial privileges. The printers met a genuine demand, urging the authors to quickly supply them with texts, sometimes with typographical errors. However, printers were accused of being solely motivated by the lure of gain. Their financial success was not to everybody's liking, notably at court, and they had to face many confrontations. Nonetheless, libel, and antiprotestant propaganda in particular, took an undeniable place in the editorial and economical choices of printer-booksellers. 